\title{
Modulation of single motor unit discharges using magnetic stimulation of the motor cortex in incomplete spinal cord injury
}

\author{
H C Smith, N J Davey, G Savic, D W Maskill, P H Ellaway, M A Jamous, H L Frankel
}

\begin{abstract}
Objectives-Motor evoked potentials (MEPs) and inhibition of voluntary contraction to transcranial magnetic stimulation (TMS) of the motor cortex have longer latencies than normal in patients with incomplete spinal cord injury (iSCI) when assessed using surface EMG. This study now examines the modulation of single motor unit discharges to TMS with the aim of improving resolution of the excitatory and inhibitory responses seen previously in surface EMG recordings.

Methods-A group of five patients with iSCI (motor level C4-C7) was compared with a group of five healthy control subjects. Single motor unit discharges were recorded with concentric needle electrodes from the first dorsal interosseus muscle during weak voluntary contraction ( $2 \%-5 \%$ maximum). TMS was applied with a $9 \mathrm{~cm}$ circular stimulating coil centred over the vertex. Modulation of single motor unit discharges was assessed using peristimulus time histograms (PSTHs).
\end{abstract}

Results-Mean (SEM) threshold (expressed as percentage of maximum stimulator output (\%MSO)) for the excitatory peak (excitation) or inhibitory trough (inhibition) in the PSTHs was higher $(p<0.05)$ in the patients (excitation $=47.1$ (5.9) \%MSO; inhibition $=44.3$ (3.2) \%MSO) than in controls (excitation=31.6 (1.2) \%MSO; inhibition=27.4 (1.0) \%MSO). Mean latencies of excitation and inhibition were longer $(p<0.05)$ in the patients (excitation=35 (1.8) $\mathrm{ms}$; inhibition=47.1 (1.8) $\mathrm{ms}$ ) than in the controls (excitation=21.1 (1.6) $\mathrm{ms}$; inhibition=27 (0.4) ms). Furthermore, the latency difference (inhibition-excitation) was longer $(\mathbf{p}<0.05)$ in the patients $(10.4(2.1) \mathrm{ms})$ than in the controls $(6.2(0.6) \mathrm{ms})$.

Conclusion-Increased thresholds and latencies of excitation and inhibition may reflect degraded corticospinal transmission in the spinal cord. However, the relatively greater increase in the latency of inhibition compared with excitation in the patients with iSCI may reflect a weak or absent early component of cortical inhibition. Such a change in cortical inhibition may relate to the restoration of useful motor function after iSCI.

(F Neurol Neurosurg Psychiatry 2000;68:516-520)

Keywords: spinal cord injury; corticospinal; motor unit; magnetic stimulation

In incomplete spinal cord injury (iSCI) motor evoked potentials (MEPs) and inhibitory responses assessed from surface EMG recordings in response to transcranial magnetic stimulation (TMS) have delayed onset latencies. ${ }^{1}$ Because of its potential relevance to rehabilitation and recovery it is important to understand whether these delays represent altered transmission in the spinal cord or reorganisation of cortical circuitry. A component of the delay may simply be slowing of conduction in the corticospinal tract as it passes through the lesion site. ${ }^{2}$ However, the inhibition was found to be proportionately more delayed than the $\mathrm{MEP}^{3}{ }^{3}$ Inhibitory responses result, at least in part, from reduced cortical output suggesting that TMS activates intracortical inhibitory neurons. ${ }^{1}$ Thus, the additional delay may be due to intracortical reorganisation in which the strength of inhibitory inputs producing the earliest component of the inhibition have become weakened. ${ }^{45}$ Similar weakening of early intracortical inhibition has been seen in schizophrenic patients taking antidopaminergic medication ${ }^{6}$ and in patients with Parkinson's disease, ${ }^{7}$ implying that dopamine may be an inhibitory transmitter involved. Our previous investigations in iSCI have been carried out using surface EMG, recording action potentials from many motor units in the target muscle. The interpretation of altered levels of EMG in response to stimuli is not straightforward. Weak or infrequent excitatory responses within the EMG of a voluntary contraction may not be rectified about a true zero and may be missed or even misinterpreted at inhibition. ${ }^{8}$ Furthermore, excitation of some motor units at different, or prolonged, latencies could obscure the inhibition of other motor units. These factors might contribute to the latency of inhibition seeming to be relatively more prolonged than the MEP in iSCI. To resolve 
this issue we have now investigated the behaviour of single motor units in response to TMS in patients with iSCI. An abstract of this work has been published. ${ }^{9}$

\section{Methods}

CONTROL SUBJECTS AND PATIENTS

With local ethical approval, we recruited five control subjects (three women, two men; aged 27-52 years) and five patients with iSCI (one woman, four men; aged 27-75 years). In four patients the spinal cord lesion was the result of traumatic injury and in the other the lesion was incurred after a subdural abscess. The lesions were located between C4 and C7 and so rostral to segments $\mathrm{C} 8$ and $\mathrm{T} 1$, the level at which the spinal nerves supplying the first dorsal interosseus muscle leave the spinal cord. Two of the patients were prescribed baclofen to alleviate muscle spasms. The time since injury ranged from 21 to 112 weeks.

NEUROLOGICAL EXAMINATION

Neurological function was assessed by a physician according to the international standards for neurological and functional classification of spinal cord injury. ${ }^{10}$ Motor function was assessed by manually testing 10 muscles on each side of the body. Specifically, the finger flexors at the distal phalanx of the middle (third) finger for level C8 and the abductor of the little (fifth) finger for level T1 were assessed and reported separately. A grade ranging from 0 to 5 was awarded for each muscle, with a grade of 0 representing no detectable contraction and a grade of 5 indicating normal muscle strength during voluntary effort. An overall motor score out of a possible 100 (100 indicating normal motor function) was derived.

Sensory function was assessed on both sides of the body for light touch (principally testing posterior columns) and pin prick (principally testing spinothalamic pathways) sensation over the 28 dermatomes from C2 to $\mathrm{S} 4 / 5$. At each level a score of 2 was given for normal sensation, 1 for impaired sensation, and 0 for no sensation. As a result, a maximum score of 112 was available for each test.

SINGLE MOTOR UNIT RECORDINGS

Recordings were made from the dominant hand of control subjects (four right, one left). In the patients the worst affected side was studied or the dominant hand if there was no neurological difference between the two sides (three right, two left). A concentric needle electrode (Medelec type NDMC25) was placed in the first dorsal interosseus muscle. Recordings were amplified $(\times 1000)$ and filtered (below $100 \mathrm{~Hz}$ and above $2000 \mathrm{~Hz}, \pm 3$ $\mathrm{dB})$. Subjects were asked to make a weak voluntary contraction (about $2 \%-5 \%$ maximum) to recruit motor unit potentials in the first dorsal interosseus muscle. Subjects were provided with auditory feedback and visual display of motor unit discharge and asked to maintain a constant frequency of discharge during the recording period. The number of motor units recorded from each subject ranged from one to nine (mean 4.6). The results presented in this paper represent the data from all 24 motor units recorded in control subjects and all 22 motor units recorded in the patients.

TRANSCRANIAL MAGNETIC STIMULATION (TMS) Transcranial magnetic stimulation was delivered to the brain using a MagStim 200 stimulator connected to a $9 \mathrm{~cm}$ circular stimulating coil centred over the vertex. The relevant orientation of the coil (A or B side up) was used to stimulate the appropriate hemisphere (left or right) for the hand targeted. ${ }^{11}$ During voluntary activation of a muscle, TMS evokes an MEP followed by a period of suppressed activity or inhibition. It is possible to evoke inhibition in the absence of a preceding MEP using a stimulus strength subthreshold for the MEP. ${ }^{1}$ The inhibition typically has a latency around $10 \mathrm{~ms}$ longer than the MEP seen at higher stimulus strength. The MEP and the inhibition can therefore be studied as two independent responses each with their own threshold. Modulation of discharge of each motor unit was studied using magnetic stimulation at a number of intensities to examine the full range of excitatory and inhibitory responses.

PERISTIMULUS TIME HISTOGRAMS (PSTHS) Data were collected using a CED 1401 and SIGAVG software (Cambridge Electronic Design). The times of occurrence of each motor unit discharge relative to the cortical magnetic stimulus ( -50 to $+150 \mathrm{~ms}$ ) were measured and a peristimulus time histogram (PSTH) was constructed using $1 \mathrm{~ms}$ bin widths. The cumulative sum (cusum) of the PSTH was derived to help provide a more accurate determination of latency of excitatory and inhibitory responses. ${ }^{12}{ }^{13}$ The cusum plots the cumulative sum of the differences of the bin counts in the PSTH from the mean bin count, starting at bin $-50 \mathrm{~ms}$ and ending at bin $+150 \mathrm{~ms}$. Groups of bins in the PSTH with counts above the mean would show an upward slope in the cusum and groups below the mean a downward slope. Onset latency of a response was assessed as the time when the bin count rose significantly above (excitation) or fell significantly below (inhibition) the mean count and was confirmed by a continuous upward or downward trend in the cusum. Threshold TMS for excitation or inhibition was the lowest stimulus intensity that resulted in a significant rise or fall from the mean bin count in the PSTH.

\section{Results}

NEUROLOGICAL TESTS

Sensory function in the patients had a mean score of $101 / 112$ (range 64-112) for light touch and 98/112 (range 64-112) for pin prick. Overall motor function had a mean score of 85/100 (range 74-93). Specifically, motor grades (scored out of 5) at level C8 were 3 (one patient), 4 (three patients), or 5 (one patient) and at level T1 4 (four patients) or 5 (one patient). 
MODULATION OF SINGLE MOTOR UNIT DISCHARGES

Figure 1 shows PSTHs together with cusums constructed from single motor unit discharges from a control subject (fig $1 \mathrm{~A}-\mathrm{B}$ ) and a patient (fig $1 \mathrm{C}-\mathrm{D}$ ) in response to TMS over the motor cortex. In the examples, threshold for excitation, seen as a peak of increased motor unit firing in the PSTH, was higher (control $40 \%$ MSO; patient $40 \% \mathrm{MSO}$ ) than threshold for inhibition (control 30\% MSO; patient 35\% MSO), seen as a trough of decreased or suspended motor unit firing. The latency of the excitation in the patient $(31 \mathrm{~ms}$, fig $1 \mathrm{C})$ was 9 $\mathrm{ms}$ longer than in the control (22 ms, fig $1 \mathrm{~A})$ and the latency of the inhibition in the patient (46 ms, fig $1 \mathrm{D}$ ) was $19 \mathrm{~ms}$ longer than in the control $(27 \mathrm{~ms}$, fig $1 \mathrm{~B})$. Thus, the difference in latency between inhibition and excitation was $10 \mathrm{~ms}$ longer in the patient $(15 \mathrm{~ms})$ than in the control (5 ms).
The average latency and threshold of excitation and inhibition measured from PSTHs across all motor units studied $(n=24$, controls; $\mathrm{n}=22$, patients) is presented in fig 2 . Threshold stimulation intensity (mean (SEM) for excitation (fig 2 A) and inhibition (fig 2 B) was significantly higher (Mann-Whitney rank sum, $\mathrm{p}<0.05$ ) in the patients (excitation=47.1 (5.9) $\%$ MSO; inhibition $=44.3$ (3.2) $\%$ MSO than in controls (excitation=31.6 (1.2) \%MSO; inhibition $=27.4$ (1.0) \%MSO.

Mean (SEM) latency of excitation (fig $2 \mathrm{C}$ ) was significantly longer $(p<0.05)$ in the patients (35 (1.8) $\mathrm{ms}$ ) than the controls (21.1 (0.6) $\mathrm{ms}$ ); mean latency of inhibition (fig $2 \mathrm{D}$ ) was longer $(\mathrm{p}<0.05)$ in the patients $(47.1(1.8)$ $\mathrm{ms})$ than in controls $(27(0.4) \mathrm{ms})$. The mean difference in inhibition-excitation latency (fig 2 E) was longer $(\mathrm{p}<0.05)$ in the patients $(10.4$ (2.1) $\mathrm{ms}$ ) than in controls $(6.2(0.6) \mathrm{ms})$.
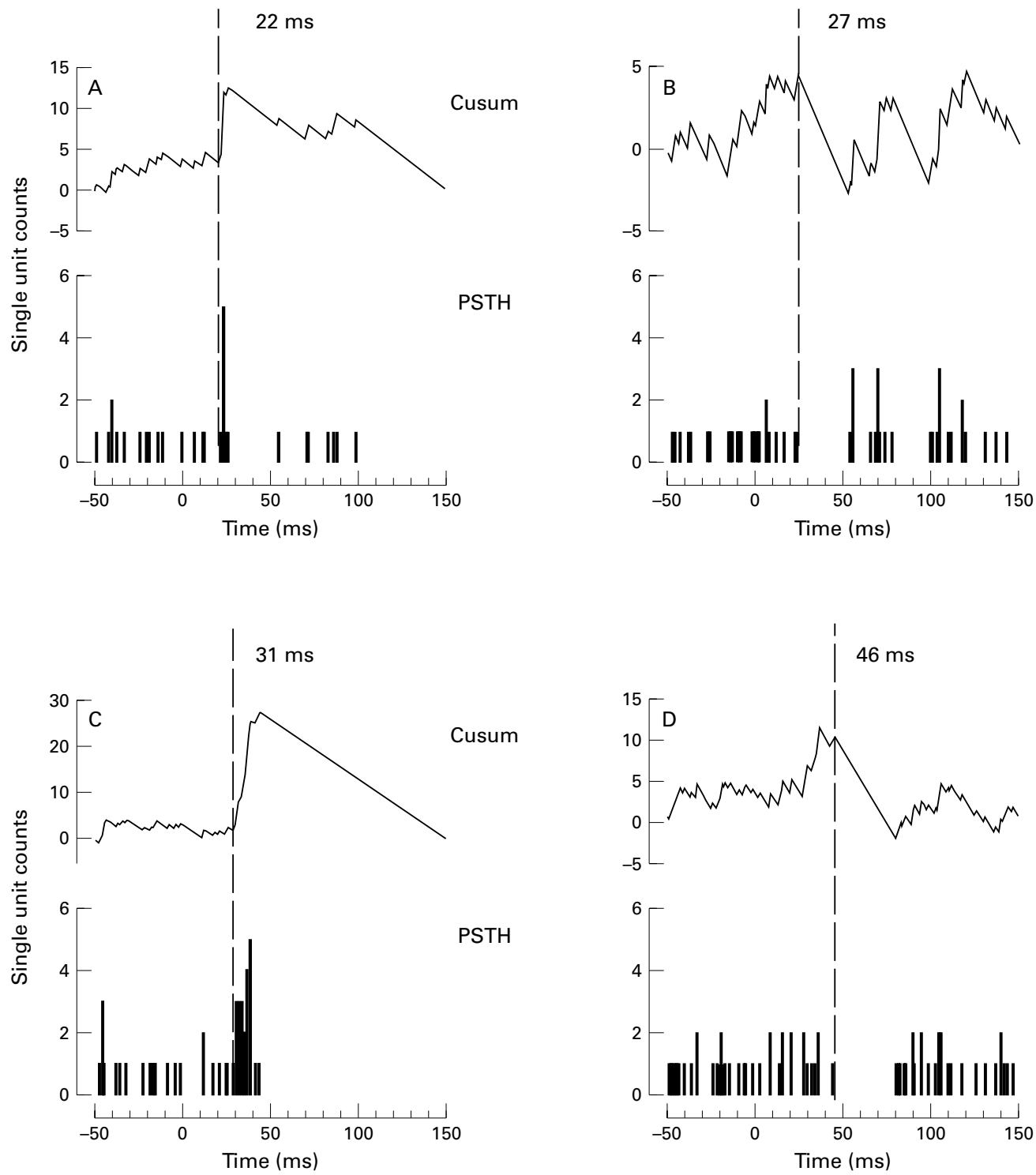

Figure 1 Single motor unit modulation in the FDI muscle to TMS of the contralateral motor cortex. (A-D) PSTHs of single motor unit modulation (bottom) with corresponding cusum (top). (A-B) Control subject (man, aged 51 years, right hand); (A) TMS at 40\% MSO, and (B) 30\% MSO. (C-D) patient with iSCI (C7 lesion, ASIA grade D, man, aged 50 years, left hand); (C) TMS at 40\% MSO, and (D) 35\% MSO. Vertical dashed lines indicate the latency of excitation (A and $C$ ) or inhibition ( $B$ and $D)$. Records constructed from the responses to 50 magnetic stimuli. 

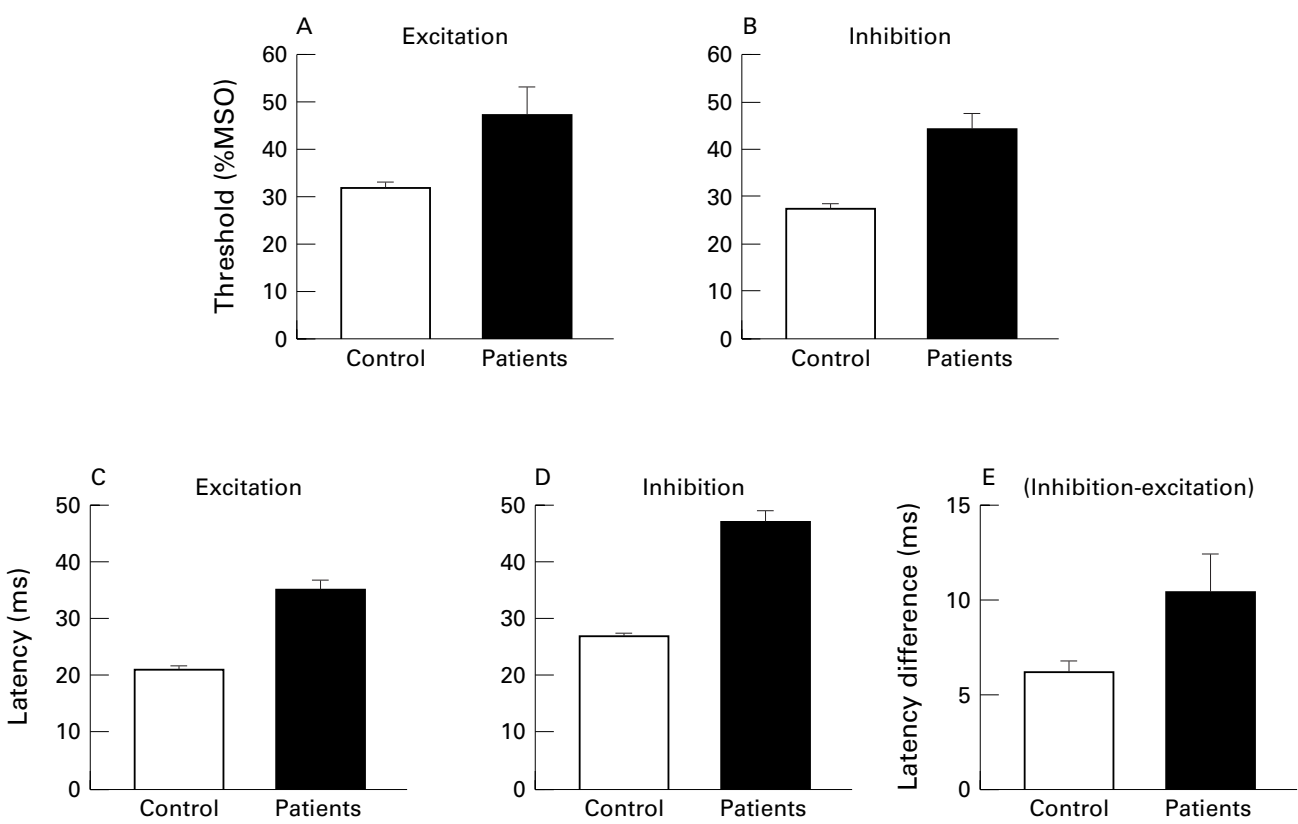

Figure 2 Thresholds and latencies of excitation and inhibition of FDI motor units to TMS applied to the contralateral motor cortex. Mean magnetic stimulation threshold for $(A)$ excitation or $(B)$ inhibition of single motor unit discharges. Mean latency of $(C)$ excitation and (D) inhibition of single motor unit discharges in patients and controls. Mean difference in latency between inhibition and excitation in (E) patients and controls. Error bars indicate 1SEM. The patients had significantly longer latencies and higher thresholds for both excitation and inhibition $(p<0.05)$. The difference in latency between inhibition and excitation was significantly longer in the patients $(p<0.05)$.

\section{Discussion}

This study shows that the discharges of single motor unit potentials in iSCI are modulated by TMS in a similar manner to gross surface EMG previously reported. ${ }^{3}$ The latency of excitatory and inhibitory modulation of single motor unit discharges in the five patients with iSCI and five controls corresponds well with the latency of MEPs and inhibitory responses from surface recordings. ${ }^{3}$ The latencies of excitation and inhibition in the control subjects are consistent with those reported by Mills and Schubert. ${ }^{14}$ These findings suggest that long latency of the inhibitory responses seen in surface recordings is a genuine feature in iSCI and not an artefact seen as a result of using multiple unit surface EMG recordings.

The neurological tests indicate that the patients have well recovered function in the muscles tested as well as good sensory and motor function generally below the lesion. These patients are neurologically and functionally similar to the group used in the study using surface EMG electrodes. ${ }^{3}$ It is therefore not unreasonable to suggest that the same aspects of the changed corticospinal physiology might have facilitated this recovery process.

The longer latency and higher stimulus threshold for the excitatory modulation of single motor unit discharges could result from several factors. Indiscriminate slowing of corticospinal neurons at the site of injury ${ }^{2}$ would lead to a widely dispersed volley from TMS of the motor cortex arriving at the motor neuron pool. Increased summation times for corticospinal volleys ${ }^{15}$ arriving at the motor neuron pool could lead to an increased latency of excitation after spinal cord injury. The dispersed nature of the descending volley at the motor neuron pool would lead to a decrease in synchronised synaptic input to the motor neurons. The overall descending volley would therefore need to be larger to achieve the same effect at the motor neuron pool, which could explain, in part, the increased excitatory threshold in iSCI. We cannot exclude the possibility that reorganisation has occurred affecting other descending pathways or at the level of the spinal motor neuron pool itself. We know from other studies that recruitment of motor neurons with increasing TMS intensity is altered in iSCI, ${ }^{16}$ which could at least in part, be due to reorganisation in the spinal motor neuron pool.

The inhibitory response to TMS seen in surface EMG recordings in normal subjects is due, in part, to a reduction of corticospinal output in response to TMS. ${ }^{1}$ Surface EMG recordings have shown that inhibition occurs at longer latency than the MEPs seen at higher stimulus intensities suggesting that a longer pathway is involved in producing the inhibition, presumably involving interneurons within the motor cortex. To dissociate latency changes resulting from slowing at the site of the lesion from more central changes, we have looked at the latency difference between inhibitory and excitatory responses. By contrast with the 6.2 (0.6) ms latency difference between the inhibition and excitation in control subjects, the patients with spinal cord injury showed a difference of 10.4 (2.1) ms. The latency difference found is therefore equivalent to an increase in latency of inhibition of $4.2 \mathrm{~ms}$ longer than might be expected from local slowing of conduction at the site of the spinal cord lesion. We have previously proposed that the inhibition comprises at least two components ${ }^{6}$ and that the early portion has become weakened or is absent after injury. ${ }^{3}$ Ridding et 
$a l^{7}$ have used paired TMS pulses to show that cortical inhibitory drive is also reduced in patients with Parkinson's disease off levodopa therapy. The largest reduction in inhibitory drive was seen using interstimulus intervals of 1-5 ms suggesting that the inhibition had become weakened during the early portion of the response. This interstimulus interval was similar to the extra difference in latency between inhibition and excitation that we found in iSCI. This work ${ }^{67}$ may then be a pointer to the inhibitory neurotransmitter dopamine being involved in production of the early part of inhibition that has become modulated as part of the recovery process after iSCI.

This study has confirmed that the differences in cortical excitation and inhibition of motor neurons seen in iSCI are evident at the level of the single motor neuron. The results support the notion that iSCI has resulted in reorganisation of neuronal circuitry in the motor cortex.

This study was supported by a project grant from the Wellcome Trust. We thank Staff Nurses Anne McCreath and Cherry Gregory for their help with recruitment of patients and clinical assistance during recording sessions. We also thank the consultants, the nursing staff, and the patients at Stoke Mandeville for their involvement in this study.

1 Davey NJ, Romaigure P, Maskill DW, et al. Suppression of voluntary motor activity revealed using transcranial magnetic stimulation of the motor cortex in man. $\mathcal{F}$ Physiol 1994;477:223-35.

2 Chang CW, Lien IN. Estimate of motor conduction in human spinal cord: slowed conduction in spinal cord human spinal cord: slowed conducti

3 Davey NJ, Smith HC, Wells E, et al. Responses of thenar muscles to transcranial magnetic stimulation of the motor muscles to transcranial magnetic stimulation of the motor
cortex in incomplete spinal cord injury patients. I Neurol
4 Puri BK, Smith HC, Cox IJ, et al. The human motor cortex following incomplete spinal cord injury: an investigation using proton magnetic resonance spectroscopy. $\mathcal{F}$ Neurol Neurosurg Psychiatry 1998;65:748-54.

5 Davey NJ, Lavoie BA, Hu M, et al. A ${ }^{11} \mathrm{C}$-flumazenil positron emission tomography (PET) study to determine the GABAergic cortical changes in the motor cortex following incomplete spinal cord injury (ISCI) in humans. Abstracts Society for Neuroscience 1999;25:603-6.

6 Davey NJ, Puri BK, Lewis HS, et al. The effects of antipsychotic medication on electromyographic responses to transcranial magnetic stimulation of the motor cortex in schizophrenia. F Neurol Neurosurg Psychiatry 1997;63:468-

7 Ridding MC, Inzelberg R, Rothwell JC. Changes in cortical excitability of motor cortical circuitry in patients with Parkinson's disease. Ann Neurol 1995;37:181-8.

8 Widmer CG, Lund JP. Evidence that peaks in EMG averages can sometimes be caused by inhibition of motoneurones. F Neurophysiol 1989;62:212-19.

9 Smith HC, Davey NJ, Maskill DW, et al. Modulation of motor unit discharge by transcranial magnetic stimulation in incomplete spinal cord injury patients. I Physiol 1996;497:104P.

10 Maynard MF Jr, Bracken MB, Creasey G, et al. International standards for neurological function classification of spinal cord injury. Spinal Cord 1997;35:266-74.

11 Day BL, Dressler D, Hess CW, et al. Direction of current in magnetic stimulating coils used for percutaneous activation of brain, spinal cord and peripheral nerve. I Physiol 1990;430:617.

12 Ellaway PH. Cumulative sum technique and its application to the analysis of peristimulus time histograms. Electroencephalogr Clinical Neurophysiol 1978;45:302-4.

13 Davey NJ, Ellaway PH, Stein RB. Statistical limits for detecting change in the cumulative sum derivative of the peristimulus time histogram. F Neurosci Meth 1986;17:15366.

14 Mills KR, Schubert M. Short-term synchronisation of human motor units and their responses to transcranial magnetic stimulation. fournal Physiol 1995;483:511-23.

15 Nakamura H, Kitagawa H, KawaguchiY, et al. Intracortical facilitation and inhibition after paired magnetic stimulation in humans under anaesthesia. Neurosci Lett 1995;199: 155-7

16 Davey NJ, Smith HC, Maskill DW, et al. Comparison of input-output patterns in the corticospinal system of normal subjects and incomplete spinal cord injured patients. Exp Brain Res 1999;127:382-90. 